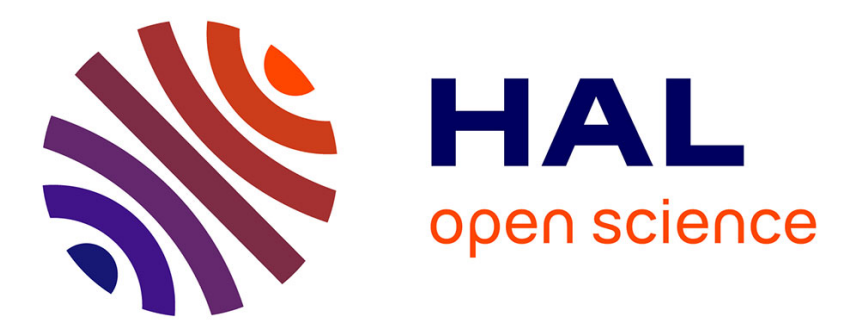

\title{
Population balance in confined comminution using a physically based probabilistic approach for polydisperse granular materials
}

\author{
C. Ovalle, C. Voivret, Christophe Dano, Pierre-Yves Hicher
}

\section{- To cite this version:}

C. Ovalle, C. Voivret, Christophe Dano, Pierre-Yves Hicher. Population balance in confined comminution using a physically based probabilistic approach for polydisperse granular materials. International Journal for Numerical and Analytical Methods in Geomechanics, 2016, 40 (17), pp.2383-2397. 10.1002/nag.2534 . hal-02007132

\section{HAL Id: hal-02007132 \\ https://hal.science/hal-02007132}

Submitted on 21 Oct 2019

HAL is a multi-disciplinary open access archive for the deposit and dissemination of scientific research documents, whether they are published or not. The documents may come from teaching and research institutions in France or abroad, or from public or private research centers.
L'archive ouverte pluridisciplinaire HAL, est destinée au dépôt et à la diffusion de documents scientifiques de niveau recherche, publiés ou non, émanant des établissements d'enseignement et de recherche français ou étrangers, des laboratoires publics ou privés. 


\title{
Population balance in confined comminution using a physically based probabilistic approach for polydisperse granular materials
}

\author{
C. Ovalle ${ }^{1, *, \dagger}$, C. Voivret ${ }^{2}$, C. Dano ${ }^{3,4}$ and P.-Y. Hicher ${ }^{3}$ \\ ${ }^{1}$ Department of Structural and Geotechnical Engineering, Pontificia Universidad Católica de Chile, Santiago, Chile \\ ${ }^{2}$ SNCF, Innovation and Research, 40 avenue des Terroirs de France, 75611 Paris, France \\ ${ }^{3}$ Ecole Centrale de Nantes, CNRS UMR, LUNAM University, Nantes 6183, France \\ ${ }^{4}$ 3SR Laboratory, CNRS UMR, University Grenoble-Alpes, Grenoble 5521, France
}

\begin{abstract}
SUMMARY
A novel, simplified approach is presented in order to compute variations of grading in granular assemblies during confined comminution under quasi-static compression. The method is based on a population balance equation and requires a breakage probability, considered here as a probabilistic phenomenon that takes into account the particle strength and the loading condition of individual grains. Under basic assumptions, a simple breakage probability can be defined in order to get a valuable result for engineering applications and powder technology. The size effect in the strength of individual particles is introduced according to Weibull's theory. The particle loading and the cushioning effect in the granular packing are accounted for by considering the orientations of the contact forces obtained from 3D discrete element method simulations of highly polydisperse materials. The method proposed could have a value for engineering purposes in powder technology and geomechanics and gives a general framework for further research developments based on population balance.
\end{abstract}

KEY WORDS: confined comminution; DEM; particle strength; polydispersity

\section{INTRODUCTION}

Comminution is the process of grain size reduction through particle fragmentation by a mechanical action. This term is usually related to grinding, a high energy consuming process used in many fields, from chemical engineering to mining, pharmaceutics, food industry, etc. In grinding mechanisms, such as ball mills, jaw crushers, etc., the material is subjected to a dynamic strike action which crushes grains and changes the grain size distribution (GSD). In such a process, the input of energy per unit of time is usually constant, and the key parameter to be optimized is time, in order to obtain a targeted GSD for a given material using a particular grinding equipment or technique. Several authors have developed methods based on population balance in each particle size fraction with the aim of predicting and optimizing this process in powder technology [1-6]. More recently, the discrete element method (DEM), used to simulate grinding comminution processes, has improved understanding of the phenomenon complexity at the micromechanical scale [7].

On the other hand, confined comminution is the grain size evolution of granular materials under quasistatic compression, also very important in powder technology and geomechanics. The prediction of grading evolution and its link to some key parameters such as porosity,

*Correspondence to: Carlos Ovalle, Department of Structural and Geotechnical Engineering, Pontificia Universidad Católica de Chile, Avda. Vicuña Mackenna 4860, Macul, Santiago, Chile.

†E-mail: covalle@ing.puc.cl 
compressibility, permeability, and critical state, is a promising area for research. This topic has been largely treated by experimental works $([8-14] \mathrm{b})$, as well as by numerical models using DEM (Åström and Herrmann, 1998; [15]). In a confined compressed granular medium, once a given intergranular contact force induces a tensile stress that exceeds particle strength, crushing develops. Then, the GSD evolves toward a more polydisperse packing, which creates a denser arrangement for a given stress condition $[8,16]$.

In our method, solid particles are considered to behave according to the Linear Elastic Brittle Mechanics Theory, and the initiation and propagation of cracks inside grains are assumed to be according to the so-called volume fracture, where transversal crack responsible of the grain fracture activates near the grain center and propagates toward the contact points [17]. It is well known that, in brittle materials, strength is inversely proportional to sample size because the probability of finding internal flaws within a specimen increases with its size [18, 19]. However, during confined comminution, small particles are more likely to break than larger ones as a result of the so-called "cushioning" or "hydrostatic" effect [15]. In other words, larger particles tend to have a higher coordination number and, therefore, are subjected to a nearly isotropic compressive stress. Other theories of grain breakage mechanics do not depend on the coordination number (i.e., Hertzian cracking that initiates at the contact); however, this type of mechanism is more likely to occur under impact loads [17], and it is not considered here.

The comprehension of the cushioning effect has been facilitated by the advances in DEM simulations, which allow to efficiently investigate the micromechanics of confined comminution [15, 20-24]. However, breakage probabilities in DEM simulations are often arbitrary, and the results have not been systematically confronted with experimental data. Moreover, when maintaining particle shape after fragmentation, the choice of the fragmentation criteria in DEM has to wrestle with pathological effects when mass conservation is imposed (i.e., increasing of local forces, excessive crushing from artificial overlaps of new fragments, creation of artificial voids after crushing, etc.) [20]. An alternative to allow fragments with shape different than the original particle is to use crushable agglomerates made by bonded spheres in a given array, assigning a breakage probability on bonds [25]. Also, recently, new methods for grain fragmentation using bonded polyhedrons have been proposed in order to avoid the limitation of creating artificial voids when particles break $[23,26]$.

We present a novel simplified approach in order to compute changes of grading in confined comminution based on a population balance equation. Our approach is based on three main hypotheses concerning the particles' strength, the breakage mechanism of particles and the contact force network:

- The particle strength has a size effect according to Weibull's theory.

- The breakage during confined comminution is mainly a result of tensile stresses within particles induced by normal inter-granular contact forces (i.e., fracture in mode I, analogous to a Brazilian test).

- For two gradings obtained after confined comminution at identical conditions but different pressure level $\left(G_{1}\right.$ and $\left.G_{2}\right)$, the inter-granular normal contact force network evolves in an analogous way as it varies for different uncrushable packings with GSDs $G_{1}$ and $G_{2}$.

Following recent works $[27,28]$, we define breakage probability by considering experimental particle strength statistics and contact forces statistically described by 3D DEM simulations of highly polydisperse uncrushable packings. Then, we analyze the orientations of the contact forces from DEM samples in order to obtain a simple but realistic description depending only on particle size for different GSDs. Thus, we are able to take into account the particle size effect and the evolution of the GSD to a more polydisperse state when crushing develops. Finally, we used published experimental data in order to verify our approach.

\section{POPULATION BALANCE IN CONFINED COMMINUTION}

Population balance models have been widely used for grading prediction during comminution in powder technology. The methodology is based on a mass balance of the $i^{\text {th }}$ material size fraction, 
where the rate of material accumulation is equal to the rate of the $i^{\text {th }}$ size production from all coarser size fractions $j$, minus the rate of breakage in fraction $i$ [6]. The following continuous form has been proposed to represent the mass distribution in batch grinding comminution [4]:

$$
\frac{d m^{i}}{d t}=\int_{j=i+1}^{\Omega} b^{i j} S^{j} d m^{j}(t)-S^{i} m^{i}(t)
$$

where $m^{i}(t)$ is the retained mass in the $i^{\text {th }}$ size fraction after a grinding time $t, S^{i}$ is the breakage probability of grains in the $i^{\text {th }}$ size fraction per unit of time, and $b^{\mathrm{ij}}$ is the fragmentation function, giving the mass contribution in the $i^{\text {th }}$ size fraction, generated by a coarser particle broken in the $j^{\text {th }}$ fraction. In Eqn (1), the size fractions are numbered from the finest to the coarsest, so for $i<j$, then $d^{i}<d^{j}$, and $\Omega$ is the coarsest fraction (where $d$ is the particle characteristic size).

$S^{i}$ and $b^{i j}$ are not necessarily derived from the knowledge of the physics of grain fracture but are empirical expressions fitted to milling data [29]. Typical functions used in comminution methods are the equations of Rosin-Rammler, Shumann and Weibull, among others [2, 4, 29]. These equations are usually expressed in terms of particle size ratios following exponential or power law functions, with one or more coefficients. Typically, the size ratio denominator is a reference size, taken as the maximal or the mean fraction, and all the other parameters are empirical. Because these expressions do not represent physical laws, their formulation can be used to model any comminution process (mills, jaw crushers, confined materials, etc.), providing the determination of suitable empirical equations and fitting parameters.

We propose the following discrete population-balance equation in order to describe the changes in each size fraction during quasistatic confined comminution:

$$
\frac{d m^{i}}{d \xi^{i}}=A^{i j} m^{i}
$$

where $d \xi^{i}$ stands for a nondimensional increment of the loading condition of grains in the $i^{\text {th }}$ size fraction, and the matrix $A^{i j}$ is defined as

$$
A^{i j}= \begin{cases}b^{i j} S^{j} & i<j \\ -S^{i} & i=j \\ 0 & i>j\end{cases}
$$

The total mass of the confined granular packing is then given by

$$
M_{\mathrm{T}}=\int_{j=1}^{\Omega} d m^{j}
$$

As well as Eqn (1), our approach is centered on $S^{i}$ and $b^{i j}$. However, to take into account the physics of the phenomena, our aim is to establish the dependence of $S^{i}$ on the characteristic particle strength $f_{f}^{i}$ and on a proper definition of $\xi^{i}$. Because we aim to describe the load applied on a particle at the micromechanical scale, a suitable choice of $\xi^{i}$ is the normalized inter-granular normal contact force $f_{n}^{i}$ (where $\langle\cdot\rangle$ stands for the mean value):

$$
\xi^{i}=f_{n}^{i} /\left\langle f_{n}^{i}\right\rangle
$$

While $f_{n}$ is defined for a contact, $f_{n}^{i}$ corresponds to a size fraction. Therefore, each $f_{n}$ contributes to two $f_{n}^{i}$. 


\subsection{Fragmentation function $b^{i j}$}

$b^{i j}$ gives the mass added to fraction $i$ from a grain breaking in fraction $j$. In other words, it describes the size of the fragments for a broken grain in the $j^{\text {th }}$ size fraction. Afterward, the evolution of the GSD for each size fraction $i$ in a granular material after comminution can be obtained by cumulating the masses given by $b^{i j}$ for all size fractions $j$ coarser than $i$, plus the mass of unbroken grains in the $i^{\text {th }}$ size fraction.

$b^{i j}$ should depend on the mineralogical and petrographic nature of the material constitutive of the grains, on their degree of degradation or weathering (i.e., internal flaws, microcracks), on the coordination number, and on the main direction of the contact forces [15]. Several authors have proposed expressions for the size distribution of fragments after rock fragmentation or soil crushing $[2,30-32]$. These expressions diverge in number of parameters and complexity but, in general, they cannot capture the physics of fragmentation. Nevertheless, empirical observations of rock fragmentation have shown that commonly, the fragments follow a self-similar distribution, which can be described by the following power law for the number of grains $N$, with size $\Delta$ coarser than $d$, where the exponent $D$ is an experimental parameter [32]:

$$
N(\Delta>d) \propto d^{-D}
$$

From Eqn (6), one can obtain the following expression for the mass of particles finer than $\Delta[33]$ :

$$
M(\Delta<d) \propto d^{3-D}-d_{\min }^{3-D}
$$

Based on the energy criterion of Griffith [18], Kendall [34] suggested that it should be impossible to break a particle below a certain size limit $d_{\text {min }}$, which might be around 1 to $10 \mu \mathrm{m}$ for rock fragments; therefore, it is reasonable to neglect $d_{\mathrm{min}}$.

Now, let us consider that the size fragment distribution from a broken particle of size $d^{j}$ follows Eqn (6). All fragments will be finer than $d^{j}$ and redistributed to finer fractions of size $d^{i}$, and the total mass of the fragments, which obviously coincides with the mass of the original particle with size $d^{j}$, is given by $M^{j}\left(\Delta<d^{j}\right) \propto\left(d^{j}\right)^{3-D}$. Similarly, in any size fraction $i$, the mass of particles finer than $d^{i}$ will be given by $M^{i}\left(\Delta<d^{i}\right) \propto\left(d^{i}\right)^{3-D}$. Therefore, the grain size cumulative distribution of all of the fragments finer than $d^{j}$ is obtained from

$$
B^{i j}=\frac{M^{i}}{M^{j}}=\left(\frac{d^{i}}{d^{j}}\right)^{3-D}
$$

Finally, for the fragmentation function $b^{i j}$, we simply compute the retained mass between two successive cumulative size fraction $(i+1$ and $i)$ :

$$
b^{i j}=B^{(i+1) j}-B^{i j}=\left(\frac{d^{i+1}}{d^{j}}\right)^{3-D}-\left(\frac{d^{i}}{d^{j}}\right)^{3-D}
$$

Eqn (9) represents a simple and versatile form with only one parameter. Of course, the global scheme of the predictive comminution method presented here will not be changed if other assumptions are made, for instance, specific fitting functions for the fragmentation of a given material.

\subsection{Breakage probability $S^{i}$}

$S^{i}$ can be obtained assuming two independent probabilistic considerations [27, 28, 35]: (i) normal compression force at breakage based on experimental particle strength statistics and (ii) a statistical description of the normal forces $f_{n}$.

2.2.1. Particle strength statistics. For particle strength characterization, we rely on the statistical theory of the strength of materials suggested by Weibull [19], which gives the following 
distribution for the survival probability of a brittle material of characteristic size $d$ subjected to a tensile stress $\sigma$ :

$$
\left.P_{S}(d, \sigma)=\exp -\left(\frac{d}{d_{\mathrm{o}}}\right)^{3}\left(\frac{\sigma}{\sigma_{\mathrm{o}}}\right)^{m}\right)
$$

where $\sigma_{\mathrm{o}}$ is the characteristic strength $\left(P_{S}\left(d_{\mathrm{o}}, \sigma_{\mathrm{o}}\right)=1 / e=0.37\right)$, and $m$ gives the data scatter and the size effect in strength. According to Jaeger [36], the characteristic tensile stress induced within the particle in a diametral compression test between two stiff parallel platens may be defined as

$$
\sigma=k \frac{f}{d^{2}}
$$

where $f$ is the diametral compression force on a particle of size $d$. The constant $k$ takes into account particle shape, for instance, Hiramatsu and Oka [37] proposed $k=0.9$, and Jaeger [36] found values of $k=1$ after crushing tests on spherical particles under diametral compression, which has been adopted here. Therefore, Weibull parameters $\left(m, d_{\mathrm{o}}\right.$, and $\left.\sigma_{\mathrm{o}}\right)$ can be obtained from a series of crushing compression tests on the particles of variable sizes [38, 39]. Then, for a constant breakage probability $\left(1-P_{S}\right)$ of $63 \%$, Eqn (10) gives the following theoretical size effect on the characteristic particle strength $\sigma_{f_{63}}^{i}$ for the $i^{\text {th }}$ size fraction:

$$
\sigma_{f_{63}}^{i}=\left(\frac{d^{i}}{d_{\mathrm{o}}}\right)^{-3 / m} \sigma_{\mathrm{o}}
$$

Finally, according to Eqn (11) (for $k=1$ ), the corresponding breakage force under diametral compression can be obtained as

$$
f_{f_{63}}^{i}=\sigma_{f_{63}}^{i} \cdot\left(d^{i}\right)^{2}
$$

The method assumes that $\sigma_{f_{63}}^{i}$ stays constant once crushing develops. However, it has to be considered that a limitation could arise for strongly heterogeneous materials when using the previous equation because breakage is likely to occur first in weakest grains and afterward $\sigma_{f_{63}}^{i}$ could increase with time.

\subsubsection{Contact forces in polydisperse materials.}

2.2.2.1. DEM simulations. In order to describe the particle loading condition at the micromechanical scale, we used the contact force network statistics based on the results of simulations of isotropic quasistatic compression on an uncrushable granular material at its denser state. To obtain a genuine description of the force network, we used the 3D DEM simulations of polydisperse frictionless uncrushable sphere packings with the Contact Dynamics Method [40] using the gdm-tk code. In order to avoid boundary effects, which are strongly enhanced in the presence of large particles, we used periodic boundary conditions without gravity [41]. The size of the smallest particle is constant $d_{\min }=0.001 \mathrm{~m}$, and $d_{\max }$ increases with the size span $R=d_{\max } / d_{\min }$, which varies from $R=1.5$ for almost monodisperse samples to 50 for highly polydisperse ones (refer to Figure 1). The GSD of the numerical samples is a power law given by the following expression

$$
F(d)=\frac{d^{-\delta+1}-d_{\min }^{-\delta+1}}{d_{\max }^{-\delta+1}-d_{\min }^{-\delta+1}}
$$

where $\delta$ varies from 2 to 3 . Figure 2 presents GSDs according to Eqn (14) for $\delta=2$ and 3, which result suitable for the DEM simulations accomplishing simultaneously two conditions: (i) high polydispersity of granular samples and (ii) relatively reduced amount of fines grains in order to avoid excessive computational cost. Despite the similarity, Eqn (14) and $b^{i j}$ should not be confused; while the first one gives grading of numerical granular packings, the second expression represents the distribution 


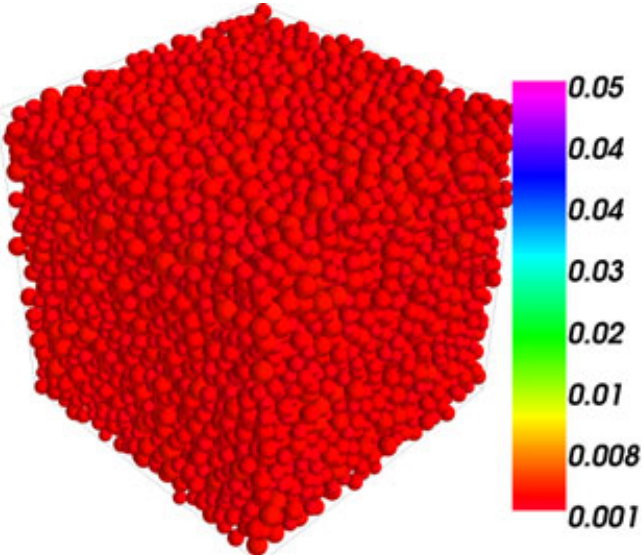

(a)

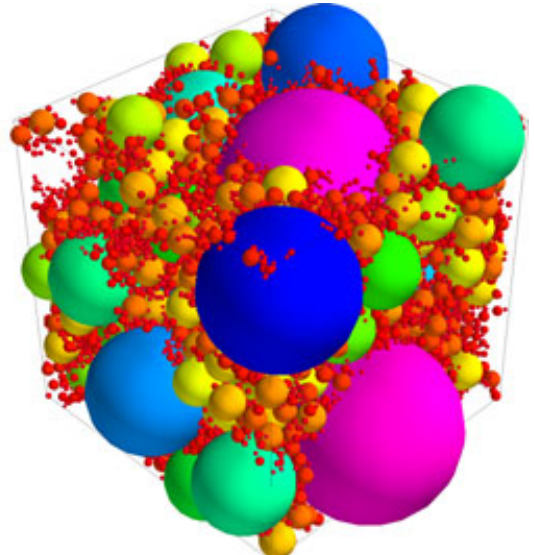

(b)

Figure 1. 3D discrete element method (DEM) samples of (a) $R=1.5$ and (b) $R=50$.

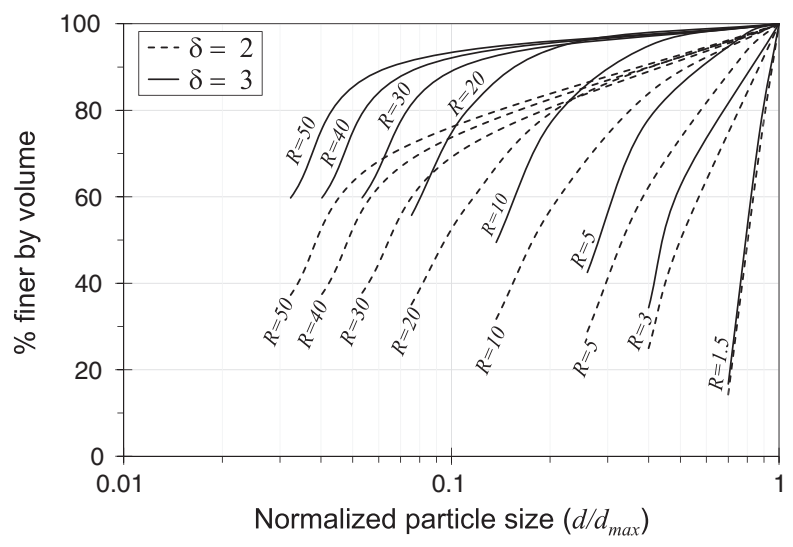

Figure 2. Grain size distribution ranges (for $\delta=2$ and 3) in DEM simulations.

of fragments of a broken grain. The building protocol of all the samples considers a first packing composed of $22^{3}$ particles of size $d_{\min }$ arranged on a square lattice. Then, the packing is subjected to an isotropic pressure and to an enlargement of each particle at a different rate, which is imposed so that the particle sizes fulfill Eqn (14) at the end of this first stage. The enlarging rate is small enough to ensure a nearly quasistatic evolution of a polydisperse random close packing. Then, the sample is allowed to relax under an isotropic effective pressure $p^{\prime}=1 \mathrm{kPa}$ until a static equilibrium state characterized by a coordination number $Z=6$ is attained. $Z$ is computed as the ratio between the number of contacts and the number of particles in the network; however, because there is no gravity in the simulations, the particles can have zero contact (floating) and then must be excluded of the computation of $Z$. Compared to a pluviated granular sample, the packing will be more isotropic in terms of fabric and so it will not have the gravity-induced features for grain contact location. At both packing and particle scales, the presence of gravity should induce anisotropies, and its magnitude should depend on the deposition protocol. On the other hand, Silbert et al. [42] showed that the friction coefficient has a small influence on the statistical distribution of contact forces for rather uniform-graded packings, and even smaller as polydispersity increases.

More details of the numerical protocol and the texture of the packings can be found in Radjai and Voivret [43] and Voivret [44].

2.2.2.2. Normal contact force network. In the polydisperse case, there are almost 10 times less contacts compared to the monodisperse sample. Hence, there is a need to increase the 
representativeness of the contact force statistics. Voivret [44] has shown that the shape of the GSD (governed only by $\delta$ in Eqn (14)) for a given size span $R=d_{\max } / d_{\min }$ has a weak influence on the probability density function (pdf) of $f_{n}$ in the 3D samples, where each $\delta$ value represents a different simulation. In other words, for the chosen GSD, $\delta$ is a second order parameter. Therefore, in order to enlarge the statistics and thus the pdf quality, we have merged the $\left\{f_{n}\right\}_{\delta}^{R}$ data along $\delta$ such that we have only one set of forces by $R$ value. Figure 3 shows the pdf of the normalized normal force group $\left\{f_{n}\right\}^{R} /\left\langle\left\{f_{n}\right\}^{R}\right\rangle$ for different values of $R$, which is independent of the stress level. It can be observed that as $R$ increases, the force distribution becomes wider.

Radjaï et al. [45] have observed that the number of $f_{n}$ lower than $\left\langle f_{n}\right\rangle$ decays as a power law, and that the number of forces higher than $\left\langle f_{n}\right\rangle$ decays exponentially. To describe the statistics of the force distribution, they proposed the following empirical expression for the pdf of the normalized value $\xi=f_{n} /\left\langle f_{n}\right\rangle$ :

$$
p_{N}(\xi)= \begin{cases}k \xi^{-\alpha} & ; \xi<1 \\ k \exp (\beta(1-\xi)) & ; \xi>1\end{cases}
$$

where $k$ is a normalizing constant $(1 / k=1 /(1-\alpha)+1 / \beta), \alpha$ and $\beta$ are empirical parameters, and the following relation can be established to ensure the continuity of the function:

$$
\beta^{2}=(1-\alpha)(2-\alpha)
$$

In order to take into account the size effects arising from particle strength and particle loading (i.e., cushioning effect), we aim to study separately the set of $f_{n}$ for each size fraction $i$. A size fraction of characteristic size $d^{i}$ can be characterized by a span factor $R^{i}=d^{i} / d_{\max }$. Now, in a given sample with size span $R$, let us consider the $i^{\text {th }}$ size fraction with size span $R^{i}$ and name this group $R \_R^{i}$. Then, every data set of normalized forces $\xi^{i}$ (refer to Eqn (5)) can be identified and regrouped in a pdf corresponding to $R_{-} R^{i}$, as shown in Figure 4. Unlike the conclusions drawn from Figure 3, we can observe in Figure 4 that the force distribution becomes narrower as $R^{i}$ increases. This can be explained by the fact that the cushioning effect on coarse grains becomes more and more dominant when polydispersity increases [44]. Moreover, Figure 5 shows that for $\xi^{i}>1$, the fitted value of $\beta$ in Eqn (15) is correlated with $R^{\mathrm{i}}$ by the following power law:

$$
\beta=1: 1149\left(R^{i}\right)^{0: 3653}
$$

This finding allows us to describe the contact force network in a simplified manner with only one parameter relying in Eqns (15) and (16), regardless of $i$ and $R$. As explained before, the numerical sample preparation considers the enlargement of each particle; therefore, $d_{\max }$ is different in each sample. In order to get comparable values of $f_{n}^{i}$, we have scaled the results in such a way that a

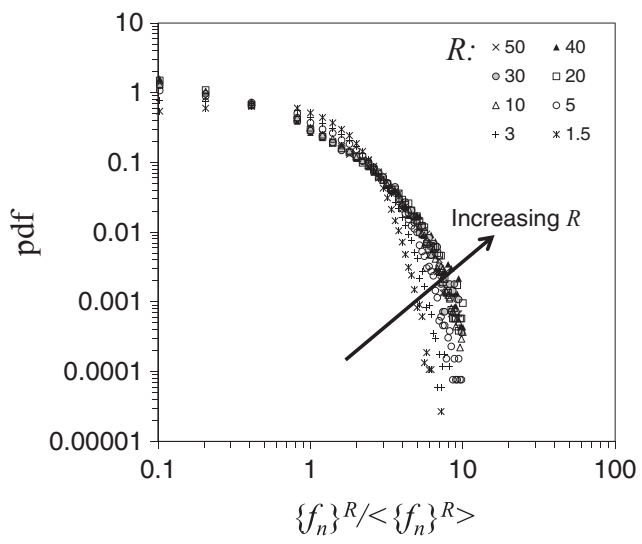

Figure 3. Probability density functions of merged normalized normal contact forces for different values of $R$. 

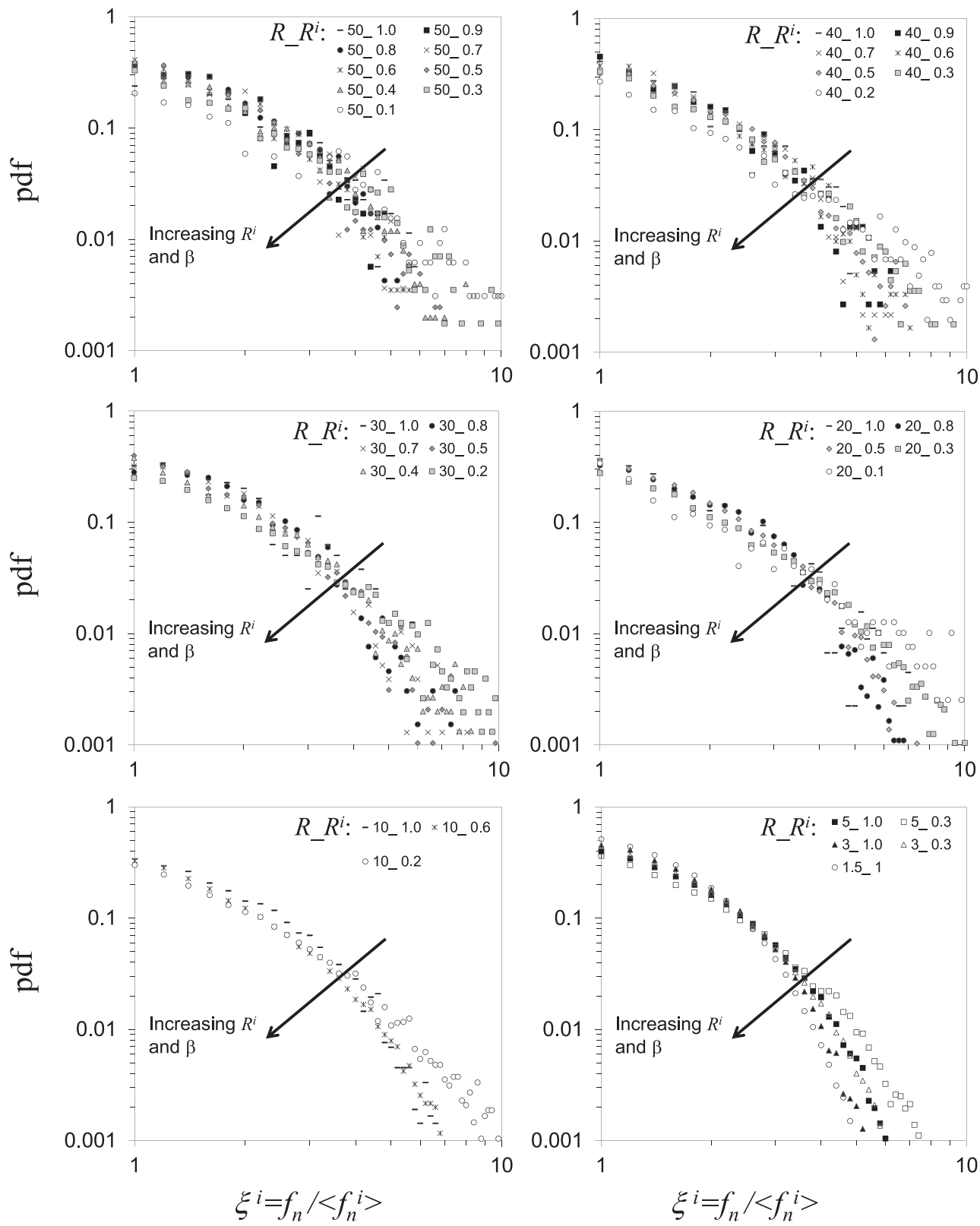

Figure 4. Probability density functions of normalized normal contact forces by size fraction $\left(R \_R^{i}\right.$ regroups the set of normalized forces in the $i^{\text {th }}$ size fraction from a sample of size span $R$ ).

constant $d_{\max }=1[\mathrm{~m}]$ for all the samples was imposed. Figure 6a presents the results as a function of $R^{i}$. It appears that except for the less dispersed case $(R=1.5)$, the samples with $R \geq 3$ are located in a very narrow band, so they can be represented by the following fitted expression (in $[\mathrm{N}]$ ):

$$
\left\langle f_{n}^{i}\right\rangle=A\left(R^{i}\right)^{2}+B\left(R^{i}\right)+C
$$

where $A=-96, B=245$, and $C=-15$ (for a correlation factor of 98\%). Data in Figure 6a mean that when $R$ increases, the contact forces are redistributed on finer particles by following a trend that does not depend on the initial $R$. For relatively low polydisperse packings, Török et al. [46] have found a linear relationship between particle size and normal force. Here, we show that this relation becomes nonlinear when polydispersity increases, and a better correlation could be obtained using a quadratic expression. 


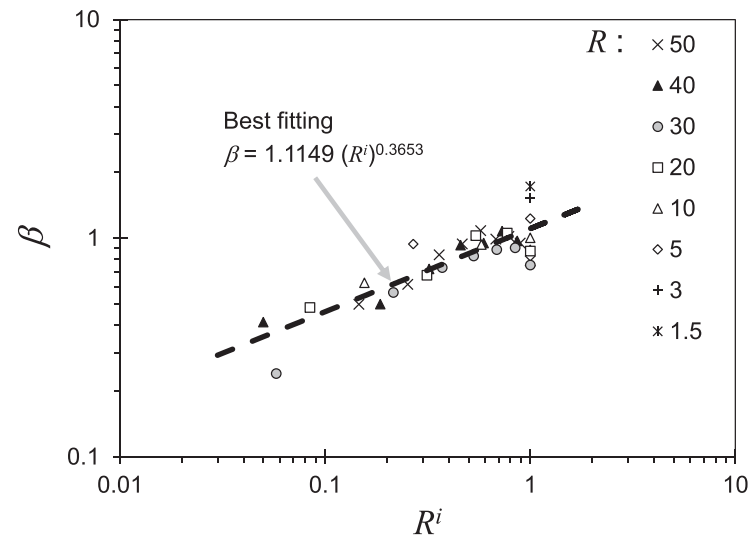

Figure 5. Fitted values for $\beta$ as a function of $R^{i}$ for several DEM samples.
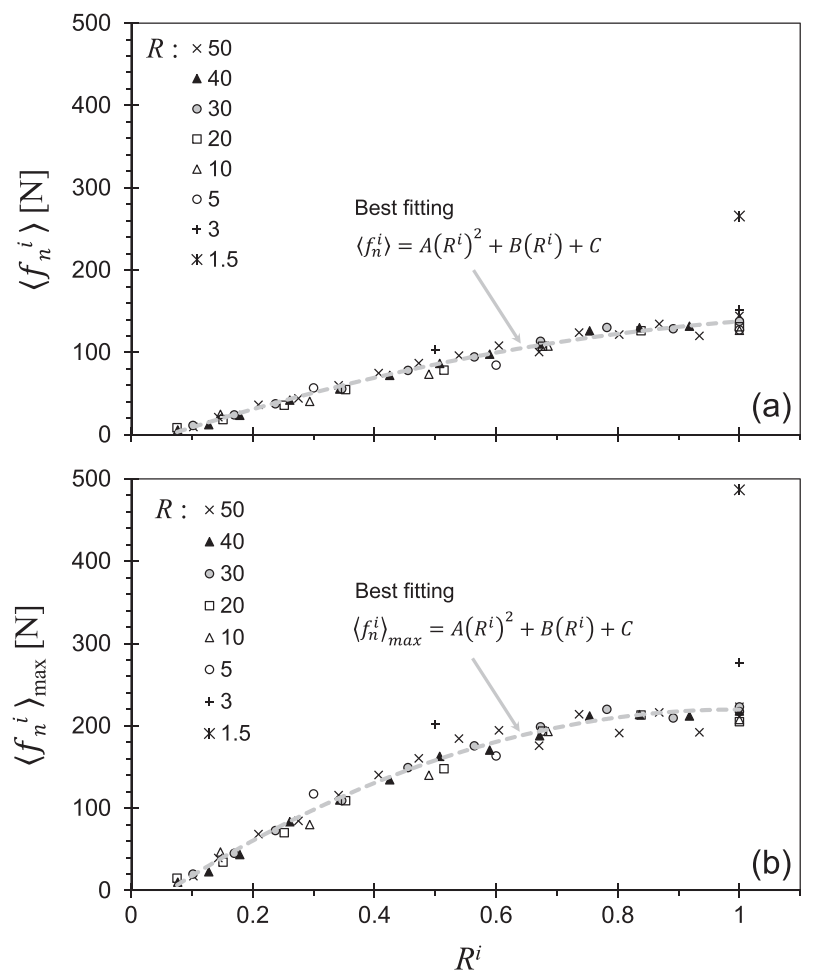

Figure 6. Average normal contact forces by size fractions for each $R$ (for a mean isotropic stress of $1000 \mathrm{~Pa}$ in DEM).

2.2.2.3. Normal force anisotropy. According to our assumption on breakage in mode I, we now attempt to distinguish between the particles subjected to highly uniaxial loading and those under rather isotropic stress, which can be obtained from a suitable definition of the force anisotropy for each particle. For all the particles in the $i^{\text {th }}$ size fraction, the orientation of $f_{n}$ can be characterized by the following fabric tensor defined by Bathurst and Rothenburg [47]:

$$
\chi_{\alpha \beta}^{i}=\frac{1}{N_{c}^{i}} \sum_{k=1}^{N_{c}^{i}} f_{n}^{k} n_{\alpha}^{k} n_{\beta}^{k}
$$

where $N_{c}^{i}$ is the number of contacts, $f_{n}^{k}$ is the normal component of the force exerted at the contact $k$, and 
$n_{\alpha}^{k}$ is the $\alpha$ component of the unit vector of the same contact. In a compressed granular packing, the normal contact forces have an average preferred orientation which often coincides with the direction of the major principal stress tensor. However, the contacts can be orientated in every direction $\theta$, and the distribution of these directions is thus characterized by a probability density [48, 49]. Azéma et al. [50] showed that the shape of the polar representation of normal contact forces could be fitted to the following harmonic approximation (refer to Figure 7):

$$
\left\langle f_{n}\right\rangle(\theta)=\left\langle f_{n}\right\rangle\left\{1+a_{n}\left(3 \cos ^{2} \theta-1\right)\right\}
$$

where the anisotropy of the normal contact forces $a_{n}$ is given by

$$
a_{n}=\frac{5}{2}\left(\chi_{1}-\chi_{3}\right) /\left\langle f_{n}\right\rangle
$$

Following this approach, for $\left\langle f_{n}^{i}\right\rangle$, we take into account the anisotropy in our comminution computations by using the maximal mean normal force in the $i^{\text {th }}$ size fraction defined for the principal direction at the particle scale as

$$
\left\langle f_{n}^{i}\right\rangle_{\max }=\left\langle f_{n}^{i}\right\rangle\left(1+2 a_{n}^{i}\right)
$$

where the anisotropy of the normal contact forces in the $i^{\text {th }}$ size fraction is given by

$$
a_{n}^{i}=\frac{5}{2}\left(\chi_{1}^{i}-\chi_{3}^{i}\right) /\left\langle f_{n}^{i}\right\rangle
$$

where the subscripts 1 and 3 refer to the principal values of the fabric tensor. Figure 8 shows that $a_{n}^{i}$ for coarse grains decreases with $R^{i}$, reflecting the cushioning effect. On the other hand, $a_{n}^{i}$ is relatively high for fine particles. Nevertheless, all the samples are located in a very narrow band, so we have simply assumed that they can be represented by the following fitted polynomial:

$$
a_{n}^{i}=1.54\left(R^{i}\right)^{3}-2.86\left(R^{i}\right)^{3}+1.35\left(R^{i}\right)+0.29
$$

As shown in Figure 6b, Eqn (18) can also be fitted for $\left\langle f_{n}^{i}\right\rangle_{\max }$, resulting in a correlation factor of $96 \%$ (for $3 \leq R \leq 50$ ) for the following parameters: $A=-253, B=502$, and $C=-30$. Finally, the cumulated probability for a contact force to be higher than the statistical value at breakage $f_{f_{63}}^{i}$ is

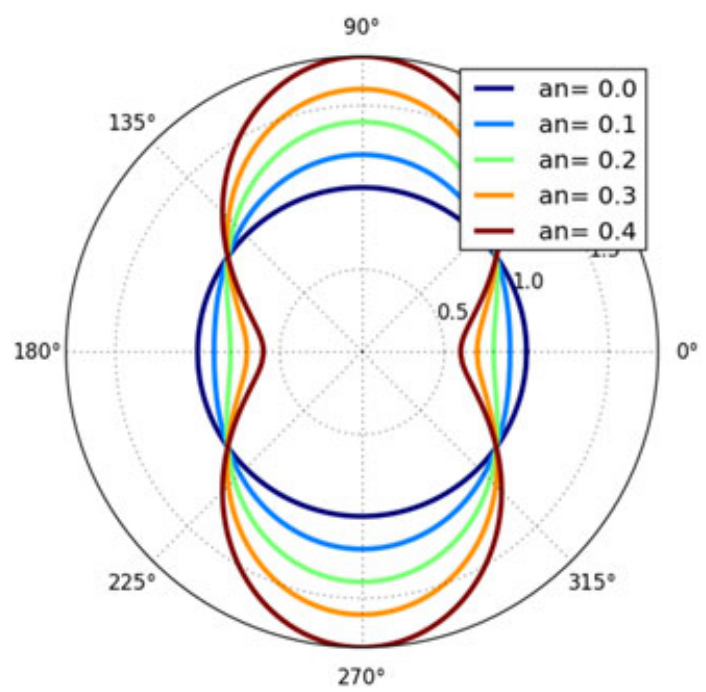

Figure 7. Shape of the polar representation of force anisotropy. 


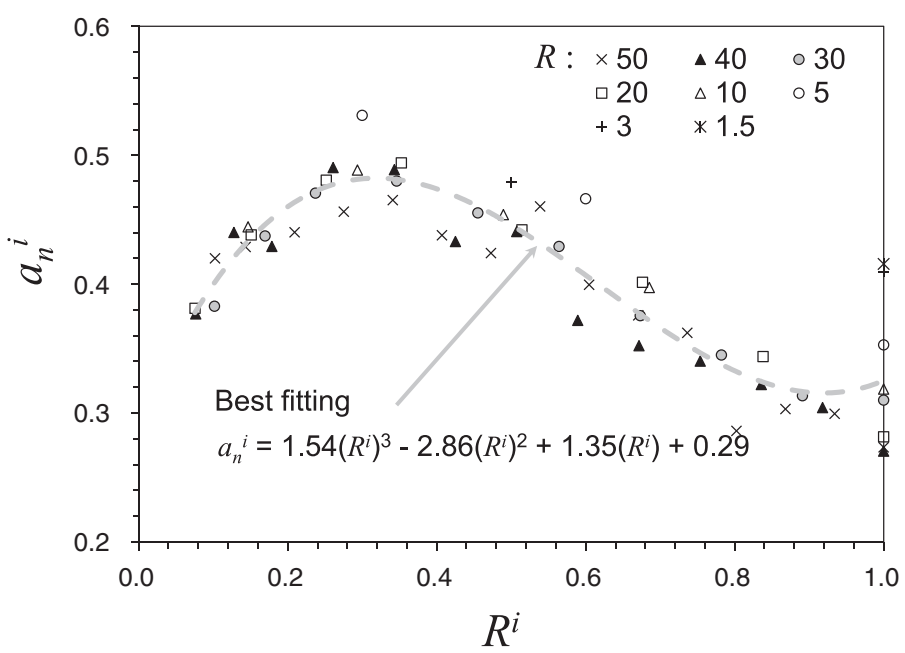

Figure 8. Anisotropy of normal contact forces by size fraction for each $R$.

given by

$$
P_{N}\left(\xi^{i}>\xi_{f_{63}}^{i}\right)=\int_{\xi_{f_{63}^{i}}^{i}}^{+\infty} p_{N}\left(\xi^{i}\right) d \xi^{i}
$$

where $\left.\xi_{f_{63}}^{i}=f_{f_{63}}^{i}=f_{n}^{i}\right\rangle_{\max }$. Accordingly, the higher the normal force anisotropy is, the higher the $\left\langle f_{n}^{i}\right\rangle_{\max }$ will be; therefore, a higher breakage probability will be computed with Eqn (25).

Consequently, for the statistical distribution of $\xi^{i}$, as well as for the evolution of $\left\langle f_{n}^{i}\right\rangle$ and $a_{n}^{i}$ with $R^{i}$, we exploit the topology of the normal contact force networks in order to describe them using expressions which are independent of $R$. This finding simplifies the confined comminution method in that it requires relatively few parameters.

2.2.3. Combined breakage probability. We assume that the force network during comminution (as well as the grading) evolves in the same way as it does in different uncrushable samples when polydispersity increases. Therefore, the breakage probability in the confined granular material is described for the $i^{\text {th }}$ size fraction by $S^{i}$, which is the combination of both assumed independent events: (i) breakage probability given by the Weibull distribution at the characteristic-induced tensile stress $\sigma_{f_{63}}^{i}$ (i.e., $P_{S}=37 \%$ ) and (ii) probability of a normalized normal contact force higher than $\xi_{f_{63}}^{i}$ :

$$
S^{i}=\left[1-P_{S}\left(d^{i} ; \sigma_{f_{63}}^{i}\right)\right] \cdot P_{N}\left(\xi^{i}>\xi_{f_{63}}^{i}\right)=0: 63 \cdot P_{N}\left(\xi^{i}>\xi_{f_{63}}^{i}\right)
$$

\section{EXPERIMENTAL VERIFICATION}

For checking how the method works, we used the experiments reported by Ezaoui et al. [51], carried out on spherical grains fabricated by mineral powder agglomeration, with a diameter from 1.6 to $4.0 \mathrm{~mm}$. The authors performed both crushing tests of several individual grains and isotropic quasistatic compression tests of granular packings, measuring the fine content (\% finer than $1.6 \mathrm{~mm}$ ) before and after each test. The tests were performed under isotropic effective stresses $p$ ' from 0.2 to 3.0 MPa. Figure 9 shows the initial GSD and the evolution of the fine content after testing. They also performed a Weibull statistical analysis of 124 individual crushing tests, obtaining the parameters in Table I from the calibration of Eqn (10). 


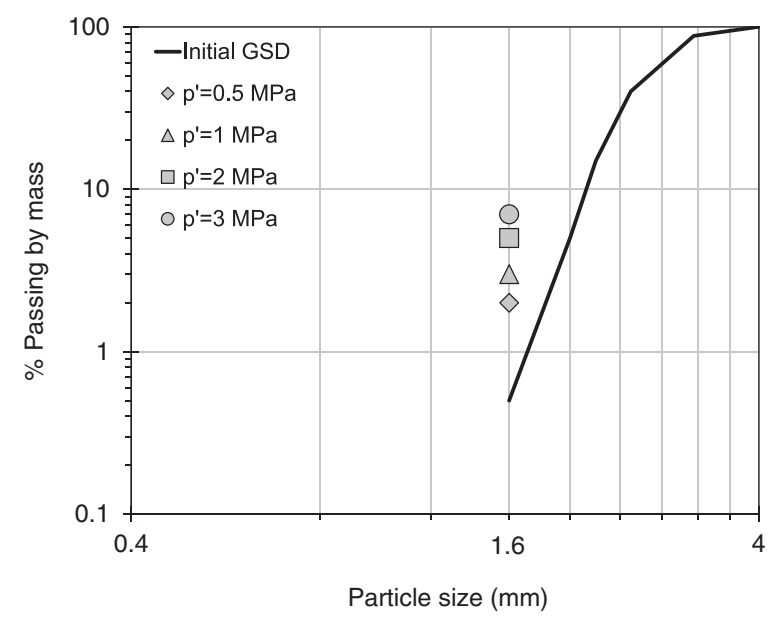

Figure 9. Initial grain size distribution and fine content (\% finer than $1.6 \mathrm{~mm}$ ) after isotropic compression tests reported by Ezaoui et al. [51].

Table I. Material properties for experimental verification.

\begin{tabular}{ll} 
Characteristic particle size $d_{\mathrm{o}}[\mathrm{mm}]$ & 3 \\
$m$ & 6.5 \\
$\sigma_{\mathrm{o}}[\mathrm{MPa}]$ & 0.91 \\
\hline
\end{tabular}

Following the work of Ovalle et al. [28], we chose a parameter $D=2.0$ for the fragmentation function (Eqn (9)). In Figure 10, we present our predictions for the fine content computed with Eqns (2), (9), and (26), establishing a good agreement with the experimental results of Ezaoui et al [51]. On these computations, there is no need for parameter calibration; we simply used Weibull parameters from Table I and $D$. The rest is coming from the normal contact force description given by DEM simulations, which is common for any other computation.

In order to study the sensitivity of $D$, Figure 11 shows the correlation factor $(\rho)$ between experimental and predicted data. It can be seen that the best fit is obtained for $D$ varying from 2.0 to 2.3. This conclusion is applicable for the purpose of this paper, which is the prediction of the fine content. However, if the aim is to predict the entire GSD, $D$ could play an important role in the shape of the curve and, therefore, it should be expected that the method would be more sensitive to its value.

Figure 10 shows also the role of the normal contact force anisotropy and the cushioning effect through $a_{n}^{i}$ in the proposed method. Because finer grains have higher force anisotropy, according to Eqn (22), the mean force is increased by a relatively higher factor. Therefore, breakage probability

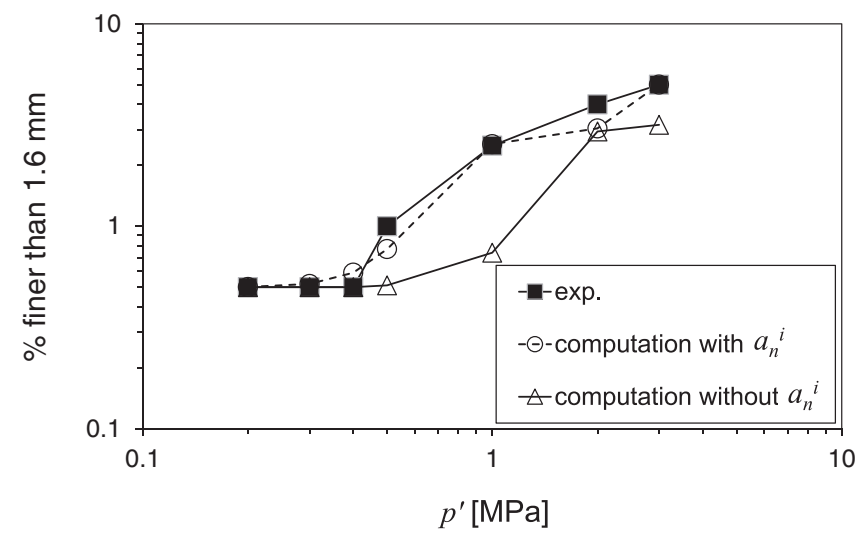

Figure 10. Prediction of fine content after confined comminution during isotropic compression. 


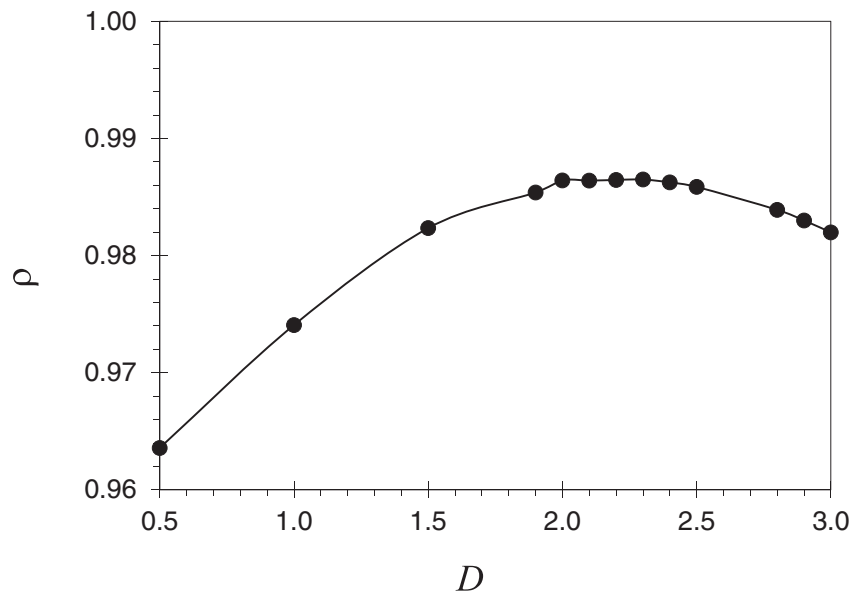

Figure 11. Correlation factor depending on $D$ between experimental and predicted values of fines content.

computed by the proposed method increased. By neglecting this effect, the predictions of crushing are too low, and the shape of the curve cannot be accurately obtained.

\section{CONCLUSIONS}

In this paper, a strategy to predict changes in grading during quasistatic-confined comminution is presented. The method is based on the population balance equation and on the probabilistic phenomenon of grain splitting in a confined granular assembly. Breakage probability is described, assuming two independent random events: (i) single particle crushing probability under uniaxial compression and (ii) probability of having a normal inter-granular contact force higher than the statistical particle strength. Firstly, particle strength statistics are described by using Weibull's theory, capturing size effects through experimental data scatter. Secondly, statistics of inter-granular forces are obtained from the 3D DEM simulations of uncrushable spherical granular packings of different grading, varying from monodisperse to highly polydisperse assemblies. Particle fracture is assumed to be caused mainly by tensile stress induced by normal contact forces. The statistical distribution of forces is shown to exhibit a consistent topology, which leads to a simple description dependent only on the particle size. This finding simplifies the confined comminution method, for it requires a relatively few number of parameters.

Using published experimental results on individual grain crushing and isotropic compression tests on granular packings, our computations appear to be in good agreement with the amount of fine content after crushing when the isotropic stress increases. These computations could have a value for engineering purposes in powder technology and geomechanics. Moreover, the method proposed gives a general framework for further developments based on the population balance equation. In this paper, simplified expressions are proposed for breakage probability; however, further research could be performed on the following main shortcomings of the method: (a) analyze the dependence interdependence between force contact network and particle strength and (b) introduce an evolution of particle strength once crushing develops, as a result of early crushing of weaker particles. At that point, computations should be fairly comparable with the full evolution of the grading in experiments.

\section{ACKNOWLEDGEMENTS}

This research work benefited from the financial support of SNCF France. Carlos Ovalle gratefully acknowledges the financial support of CONICYT Chile through the project FONDECYT 11150084, Vicerrectoría Académica de Investigación VRI of Pontificia Universidad Católica de Chile through projects PIA 2015 3519-301 and VRI Inicio 3514-804. 


\section{REFERENCES}

1. Austin LG. A discussion of equations for the analysis of batch grinding data. Powder Technology 1999; 106:71-77.

2. Austin LG, Shoji K, Bhatia V, Jindal V, Savage K. Some results on the description of size reduction as rate process in various mills. Industrial and Engineering Chemistry Process Design and Development 1976; 15(1):187-197.

3. Bass Z. On the theory of grinding. Journal of Applied Mathematics and Physics 1954; 5(4):283-292.

4. Das PK, Khan AA, Pitchumani B. Solution to batch grinding equation. Powder Technology 1995; 85:189-192.

5. Epstein B. Logarithmico-normal distribution in breakage of solids. Industrial and Engineering Chemistry 1948; 40(12):2289-2291.

6. Reid KJ. A solution to the batch grinding equation. Chemical Engineering Science 1965; 20:953-963.

7. Weerasekara NS, Powell MS, Cleary PW, Tavares LM, Evertsson M, Morrison RD, Quist J, Carvalho RM. The contribution of DEM to the science of comminution. Powder Technology 2013; 248:3-24.

8. Biarez J, Hicher PY. Influence de la granulométrie et de son évolution par ruptures de grains sur le comportement mécanique de matériaux granulaires. Revue Française de Génie Civil 1997; 1(4):607-631.

9. Biarez J, Hicher PY. Elementary Mechanics of Soil Behaviour. Balkema: Rotterdam, 1994.

10. Lade P, Yamamuro J, Bopp P. Significance of particle crushing in granular materials. Journal of Geotechnical Engineering 1996; 122(4):309-316.

11. Lee I, Coop M. The intrinsic behaviour of a decomposed granite soil. Geotechnique 1995; 45(1):117-130.

12. Ovalle C, Dano C, Hicher P-Y. Experimental data highlighting the role of surface fracture energy in quasi-static confined comminution. International Journal of Fracture 2013a; 182(1):123-130.

13. Ovalle C, Dano C, Hicher P-Y, Cisternas M. Experimental framework for evaluating the mechanical behavior of dry and wet crushable granular materials based on the particle breakage ratio. Canadian Geotechnical Journal 2014a; 52(5):587-598.

14. Vesic AS, Clough GW. Behaviour of granular materials under high stresses. Journal of the Soil Mechanics and Foundations Division ASCE 1968; 94(SM 3):661-688.

15. Tsoungui O, Vallet D, Charmet JC. Numerical model of crushing of grains inside two-dimensional granular materials. Powder Technology 1999a; 105:190-198.

16. Li G, Ovalle C, Dano C, Hicher P-Y. Influence of grain size distribution on critical states of granular materials. In Constitutive Modeling of Geomaterials. Springer Series in Geomechanics and Geoengineering, Yang Q et al. (eds.). Springer: Berlin, 2013; 207-210.

17. Tsoungui O, Vallet D, Charmet JC, Roux S. Size effects in single grain fragmentation. Granular Matter 1999b; 2(19):19-27.

18. Griffith AA. The phenomena of rupture and flow in solids. Philosophical Transactions of the Royal Society A: Mathematical, Physical and Engineering Sciences 1921; 221:163-198.

19. Weibull W. A statistical theory of the strength of materials. Proceedings of Royal Swedish Institute of Engineering Research 1939; 151:1-45.

20. Åström JA, Herrmann HJ. Fragmentation of grains in a two dimensional packing. The European Physical Journal B 1998; 5:551-554.

21. Ben-Nun O, Einav I, Tordesillas A. Force attractor in confined comminution of granular materials. Physical Review Letters 2010; 104:108001.

22. De Bono JP, McDowell GR. DEM of triaxial tests on crushable sand. Granular Matter 2014; 16:551-562.

23. Nguyen D-H, Azéma E, Sornay P, Radjai F. Bonded-cell model for particle fracture. Physical Review E 2015; 91:022203.

24. Yang Y, Cheng YM. A fractal model of contact force distribution and the unified coordination distribution for crushable granular materials under confined compression. Powder Technology 2015; 279:1-9.

25. Cheng Y, Nakata Y, Bolton MD. Discrete element simulation of crushable soil. Geotechnique 2003; 53(7):633-641.

26. Cantor D, Estrada N, Azéma E. Split-cell method for grain fragmentation. Computers and Geotechnics 2015; 67:150-156.

27. Marketos G, Bolton MD. Quantifying the extent of crushing in granular materials: a probability-based predictive method. Journal of the Mechanics and Physics of Solids 2007; 55:2142-2156.

28. Ovalle C, Voivret C, Dano C, Hicher P-Y.A probabilistic approach of confined comminution in polydisperse granular materials. Powders and Grains, July 8-12 2013, Sydney, Australia. AIP Conf. Proc. 1542, 2013b; pp. $903-906$.

29. Perry RH, Green D. Perry's Chemical Engineers' Handbook (7th). McGraw-Hill: New York, 1997.

30. Fukumoto T. A grading equation for decomposed granite soil. Soils and Foundations 1990; 30(1):27-34.

31. Ocampo M, Caicedo B. Modeling of abrasion and crushing of unbound granular materials during compaction. Powders and Grains, July 13-17 2009, Golden, U.S.A.. AIP Conf. Proc. 1145, 2009; pp. 855-858.

32. Turcotte D. Fractals and fragmentation. Journal of Geophysical Research 1986; 91(B2):1921-1926.

33. Einav I. Breakage mechanics — part I: theory. Journal of the Mechanics and Physics of Solids 2007; 55(6):1274-1297.

34. Kendall K. The impossibility of comminuting small particles by compression. Nature 1978; 279:710-711.

35. Zhou B, Wang J, Wang H. A new probabilistic approach for predicting particle crushing in one-dimensional compression of granular soil. Soils and Foundations 2014; 54(4):833-844.

36. Jaeger JC. Failure of rocks under tensile conditions. International Journal of Rock Mechanics and Mining Sciences 1967; 4:219-227.

37. Hiramatsu Y, Oka Y. Determination of the tensile strength of rock by a compression test of an irregular test piece. International Journal of Rock Mechanics and Mining Sciences 1966; 3(2):89-90. 
38. McDowell GR, Amon A. The application of Weibull statistics to the fracture of soil particles. Soils and Foundations 2000; 40(5):133-141.

39. Ovalle C, Frossard E, Dano C, Hu W, Maiolino S, Hicher P-Y. The effect of size on the strength of coarse rock aggregates and large rockfill samples through experimental data. Acta Mechanica 2014b; 225:2199-2216.

40. Moreau J. In Vol 14 of Lectures Notes in Applied and Computational Mechanics. Novel Approaches in Civil Engineering, Vol. 2004, Frémond M, Maceri F (eds.). Springer: Berlin; New York, 2004; 1-46.

41. Voivret C, Radjaï F, Delenne J-Y, El Youssoufi MS. Multiscale force networks in highly polydisperse granular media. Physical Review Letters 2009; 102:178001.

42. Silbert L, Grest G, Landry J. Statistics of the contact network in frictional and frictionless granular packings. Physical Review E 2002b; 66:061303.

43. Radjai F, Voivret C. Periodic boundary conditions. In Discrete Numerical Modeling of Granular Materials, Radjaï F, Dubois F (eds.). Wiley-Iste: London, United Kingdom, 2011; 181-198.

44. Voivret C. Cushioning effect in highly polydisperse granular media. Powders \& Grains 2013, July 8-12 2013, Sydney, Australia. AIP Conf. Proc. 1542, 2013; 405.

45. Radjaï F, Jean M, Moreau JJ, Roux S. Force distributions in dense two-dimensional granular systems. Physical Review Letters 1996; 77(2):274-277.

46. Török J, Unger T, Fazekas S, Wolf D. Relationship between particle size and normal force. In Powders and Grains 2005, García-Rojo R, Herrmann HJ, McNamara S (eds.). Balkema: Rotterdam, 2005; 1273-1277.

47. Bathurst RJ, Rothenburg L. Micromechanical aspects of isotropic granular assemblies with linear contact interactions. Journal of Applied Mechanics 1988; 55(1):17-23.

48. Oda M, Iwashita K. Mechanics of Granular Materials. A. A. Balkema: Rotterdam, 1999.

49. Satake M. Fabric tensor in granular materials. In Proceedings of the IUTAM Symposium on Deformation and Failure of Granular Materials, Vermeer PA, Luger HJ (eds.). Balkema: Delft, Amsterdam, 1982; 63-68.

50. Azéma E, Radjai F, Saussine G. Quasistatic rheology, force transmission and fabric properties of a packing of irregular polyhedral particles. Mechanics of Materials 2009; 41:729-741.

51. Ezaoui A, Lecompte T, Di Benedetto H, Garcia E. Effects of various loading stress paths on the stress-strain properties and on crushability of an industrial soft granular material. Granular Matter 2011; 13(4):283-301. 